Original Research Paper

\title{
Contributions to the Hydrogen Fusion
}

\author{
Relly Victoria Virgil Petrescu and Florian Ion Tiberiu Petrescu
}

ARoTMM-IFToMM, Bucharest Polytechnic University, Bucharest, (CE), Romania

\author{
Article history \\ Received: $15-08-2020$ \\ Revised: 26-08-2020 \\ Accepted: 28-08-2020 \\ Corresponding Author: \\ Relly Victoria Virgil Petrescu \\ ARoTMM-IFToMM, \\ Bucharest Polytechnic \\ University, Bucharest, (CE), \\ Romania \\ Email: rvvpetrescu@gmail.com
}

\begin{abstract}
The issue of hydrogen fusion is an important one because once done it will generate enough energy for humanity, which is why the paper addresses some new ideas in this important but also necessary field. Attempts are made to make theoretical considerations modeled so that it can then help experimentally to put into practice the nuclear fusion of hydrogen, in its most elementary and simplest form, between hydrogen protons, without using deuterium as fuel, but directly ionized hydrogen atoms, protons, so only the first isotope of hydrogen, this reaction being in a way the most natural, it taking place permanently in the stars, including our sun. The paper brings some new theoretical ideas including the possibility of accelerating protons before their collision to energy capable of fusion, the introduction of neutrons with protons in the fusion tank because the energy required for proton-neutron fusion is slightly lower (as will be possible observed in the paper), or the production of neutrons directly from the existing protons so that the nuclear fusion reaction becomes easier between proton-neutron (by bombarding protons with accelerated electrons).
\end{abstract}

Keywords: Fusion, Hydrogen, Proton, Neutron, Electron, Nuclear Energy, A Down Quark, An Up Quark, Nuclear Fusion, Hydrogen Proton, Kinetic Energy

\section{Introduction}

To produce electricity, a heat source is needed to heat the water in order to obtain steam under pressure. These vapors expand into a steam turbine that drives an alternating current generator that produces electricity.

Such a process of obtaining electricity also takes place in nuclear power plants (CEN) - also called nuclear power plants or nuclear power plants.

If the heat resulting from the condensation of vapors is recovered and used for heating, the notion of cogeneration appears.

Atomic or nuclear energy is the energy released by atomic nuclei following the process of FISSION or FUSION, in significant quantities.

The contribution of nuclear energy in 2010 was $50 \%$ of total energy production. It is estimated that by 2020 , nuclear energy will account for $57 \%$ of the total energy produced globally. This is explained by the possibility of using currently available nuclear technologies - type Generation III, which incorporates the latest security requirements. A series of Generation IV technologies are being designed, which will become available starting with 2020 .

Some security and cost performance:
- Projected lifespan: $40 \ldots 60$ years

- Availability greater than $90 \%$

- Optimization of combustion and recharging - cycles of 12-24 months

- Low probability of significant releases of radioactivity in the active area and outside the site

- Nuclear electricity production is the only one that, during the technological process, practically does not eliminate toxic waste in the environment

In Europe, France is the largest producer of nuclear energy: $73.2 \%$ of total energy production, almost 340,000 million $\mathrm{kWh}$ in 2000.

Studies have shown that nuclear energy can provide exclusively the energy needed by the world's population, with minimal effects on the environment.

Nuclear energy contributes to the fulfillment of the fundamental objectives of energy policy: (1) -Energy security; (2) -Saving resources; (3) -Environmental protection.

Today, nuclear energy is released by atomic nuclei, in huge quantities, through the process of nuclear fission, but it is also intensely prepared for nuclear fusion.

Nuclear fission (atomic fission) achieves a division of a heavy atomic nucleus into two fragments of almost 
the same mass. Cleavage is accompanied by the release of a huge amount of energy (the binding energy of subatomic particles).

In the process of nuclear fission, the nucleus Uranium$235\left(\mathrm{U}^{-235}\right)$ combines with a neutron (n), forming an unstable intermediate element, of approximately equal mass - Uranium-236 ( $\left.\mathrm{U}^{-236}\right)$, which immediately splits into barium -144 $\left(\mathrm{Ba}^{-144}\right)$, Krypton-89 $\left(\mathrm{Kr}^{-89}\right)$ and three fast neutrons $(n 1, n 2, n 3), \gamma$ radiation and thermal energy- $E=$ 200MV. In practice, most of this caloric energy occurs as kinetic energy because the resulting nuclei repel and move away from each other at very high speeds.

The three neutrons can bombard other nuclei, leading to other fission reactions, the process being called a chain reaction. This process allows the fission to be primed and controlled.

The energy released in the nuclear fission process is used to produce electricity for industrial and household consumers.

To the same extent, however, it can be used for propulsion ships or as a source of destructive power for nuclear weapons.

Uranium (U) is the basic fuel in nuclear energy, being a radioactive element that has two fissile isotopes ( $\mathrm{U}^{-235}$ and $\left.\mathrm{U}^{-233}\right)$ and two fertile $\left(\mathrm{U}^{-238}\right.$ and $\left.\mathrm{U}^{-234}\right)$.

One of the main ideas of this paper is the use of neutrons in nuclear fusion because they are the main pawns and craftsmen of the nuclear fission reaction.

Nuclear fusion is a process by which, following reactions between light elements - deuterium and tritium heavy elements are obtained, with the release of an immense amount of energy. Deuterium or heavy hydrogen is an isotope of hydrogen whose chemical symbol is ${ }_{1}^{2} \mathrm{H}$ or $\mathrm{D}$, atomic number 1 (atomic mass approx. 2), its nucleus being composed of a proton and a neutron.

Deuterium is a stable substance found in about $0.015 \%$ of ordinary hydrogen. It can be purified by distillation from hydrogen or can be obtained by electrolysis of water. Like ordinary hydrogen, it enters the same type of chemical reactions and forms $\mathrm{D}_{2}$ (like $\mathrm{H}_{2}$ ) and $\mathrm{D}_{2} \mathrm{O}$ (like $\mathrm{H}_{2} \mathrm{O}$ ) - heavy water.

Tritium is an isotope of hydrogen heavier than deuterium, its nucleus consisting of a proton and two neutrons. Tritium is obtained from lithium - a light metal.

Nuclear fusion of deuterium and tritium atoms at high temperatures releases very large amounts of energy. Nuclear fusion is the most present energy process in the Sun and stars. The energy released by the Sun and other stars is the result of fusion reactions between hydrogen nuclei. Such nuclear fusion reactions underlie the operation of experimental nuclear power reactors, as well as the operation of nuclear weapons hydrogen. The hydrogen bomb works by inducing fusion reactions in a mixture of hydrogen isotopes - deuterium and tritium, obtaining a heavy helium nucleus.
Nuclear fusion is difficult to produce artificially and very difficult to control. If you get a controlled nuclear reaction, it can be a very cheap source of energy because deuterium - the main element in the fusion reaction, can be extracted from ordinary water. An amount of energy equivalent to that produced by 9,450 liters of gasoline can be produced from 30 liters of water.

The practical realization of a fusion reactor will lead to the possibility of humanity to benefit in the future from unlimited energy resources. The energy obtained from hydrogen is inexhaustible and accessible practically all over the globe. For the processing and maintenance of a nuclear fusion reaction, the two nuclei (deuterium and tritium) that fuse must lose their electrons and be accelerated to particularly high speeds.

In order to prevent the mutual rejection of the two positively charged nuclei, the temperature of the particles is raised to values several times higher than the temperature at the surface of the Sun.

In practice, this temperature becomes so high that the particles pass from their natural state of gaseous aggregation to another state - PLASMA.

The control, storage and isolation of plasma in a closed and safe space is the biggest problem now encountered in nuclear fusion experiments.

Use of a very strong magnetic field to protect the materials inside the fusion reactor and to prevent plasma leakage, as well as the use of a type of inertial control based on maintaining plasma cohesion by bombardment with multiple laser beams of the reactor chamber, today represent the modern methods of plasma control during the nuclear fusion reaction.

In a mass of heavy hydrogen (gas), the isotopes of deuterium and tritium fuse, releasing about 17.6 MV on a fusion element. Deuterium has an extra neutron and tritium two. In a fusion nuclear reactor, the emitted neutrons (which are responsible for $80 \%$ of the final energy produced) are absorbed into a "sheath" that surrounds the reactor core. It contains Lithium (Li) which is converted to Tritium (T) and Helium (He).

The "jacket" of the reactor is thick enough (approx. $1 \mathrm{~m}$ ) to slow down neutrons with $14 \mathrm{MeV}$ energies. This process of moderating neutron kinetics thus heats the mantle so that the presence of coolant is necessary in order to take the amount of heat released and to transfer it to a steam generation system which, in turn, produces electricity.

A second basic idea of this paper is the use of accelerated neutrons to produce additional energy by fusing them (due to their collision) with accelerated protons in turn.

In the reactor core, fusion reactions take place at temperatures exceeding 100 million degrees Celsius. The helium produced heats the new fuel and, after cooling, is extracted from the core. 
Neutrons pass through the walls of the "mantle" and interact with Lithium, thus leading to a new process of Tritium reproduction.

Fusion nuclear power plants consume less fuel and produce less waste at the same amount of energy produced.

The range of the static nucleus ranges from about $1 \times 10^{-15} \mathrm{~m}$ for hydrogen to about $7 \times 10^{-15} \mathrm{~m}$ for the heaviest known atom. Also under these conditions the outer diameter of the atom (outer electron cloud) is in the range of $1-3 \times 10^{-10} \mathrm{~m}$, that is, approximately $10^{5}$ times the diameter of the nucleus. Static so-called measurements are made at low atomic or nuclear velocities. In reality, when a nucleus moves at a higher speed, it changes its dimensions, a change that can be significant depending on its linear displacement speed v.

Nuclear fusion is the combination of two light nuclei into a heavier nucleus. The fusion or thermonuclear reaction of light elements are typical reactions that take place in the Sun and other stars. Indeed, in the Sun, every second, 657 million tons of hydrogen are converted into 653 million tons of helium. The missing 4 million tons are then converted into radiation - this phenomenon ensuring the brightness of the Sun. Extreme temperatures and high pressure create a state of matter, strongly ionized, called plasma and which is maintained in that volume by gravitational forces.

A fusion reaction in which a relatively large amount of energy (27.7 MeV) is released is one in which four protons interact leading to the formation of a helium nucleus (an alpha particle). Because hydrogen isotopes are used in this process and hydrogen is practically all around us, the idea of obtaining energy from its fusion is extremely attractive: It basically provides an unlimited source of energy for future generations!

Fusion reactions, however, are not easy to accomplish on Earth. It must be borne in mind that the required temperatures are extremely high, generally in the order of hundreds of millions of degrees Kelvin. and once the hot plasma is created, the problem of maintaining it remains, which is not an easy one.

To initiate the fusion reaction, the Coulomb repulsion between the nuclei must be defeated. Thus they must have high incident kinetic energies from a few $\mathrm{keV}$ to a few hundred $\mathrm{keV}$ (we neglect here the possibility of initiating fusion at low temperatures through the so-called meson-catalyzed fusion). It is relatively simple to accelerate light particles to these energies. However, the energy required for the operation of particle accelerators far exceeds the energy accumulated in the fusion process. It is more efficient to use an alternative solution: The kinetic energy of the reactants can occur as a result of the high temperature of a particulate gas. At temperatures of tens or hundreds of millions of degrees Kelvin, electrons are expelled from atoms, the reactants existing as a hot plasma. This is why we talk about "thermonuclear" reactions.

The main technical problem is related to the generation of extremely high temperatures and pressures in the ionized gas - plasma - and its isolation for a long enough time, so as to trigger the release of energy. Once this is done and sufficient fusion reactions take place, the conditions are self-sustaining so that continuous energy production can be achieved.

The condition for the release of energy from a thermonuclear reactor is given by Lawson's criterion that the product between the density of plasma nuclei and the time of confinement at the ignition temperature must exceed a certain threshold value.

The need to have a high temperature means that the plasma does not come into contact with the walls. Therefore, special plasma isolation techniques must be used.

There are three methods of isolating plasma: Gravitational, magnetic and inertial. In stars, the insulation is due to gravity, which creates sufficiently high pressure. This type of isolation cannot be applied on Earth. Instead, strong magnetic fields can be used to capture plasma by the magnetic confinement method or the inertial confinement method by compressing hydrogen granules with a powerful laser or a particle beam.

In the case of magnetic confinement, where the particle density is higher, about $10^{20} / \mathrm{m}^{3}$, the confinement time given by the Lawson criterion must be greater than $1 \mathrm{~s}$. In the case of inertial confinement, the typical density is $10^{31} / \mathrm{m}^{3}$ and the confinement time must be of the order of $10^{-11} \mathrm{~s}$.

Deuterium can be easily obtained from water (30 grams per cubic meter). Tritium must be produced in either a nuclear reactor or a lithium fusion reactor, an element that can be found in the earth's crust in large quantities.

This can be achieved by making a thick layer of lithium (about $1 \mathrm{~m}$ ), which also contains beryllium and which surrounds the reactor core. Lithium will absorb neutrons that are slowed down in this layer and turn into tritium and helium. The energy thus released heats the blanket, thus starting to generate conventional energy. The use of beryllium is motivated by maintaining a sufficient number of neutrons in the system.

The motion of the charged particles in the plasma can be controlled by an external magnetic field. In systems with magnetic confinement, called Tokamak reactors, plasma (D-T for example) is heated and confined to a density of about $10^{21}$ particles per cubic meter. The magnetic field is designed so that the particles remain inside the enclosure, otherwise, the temperature would drop below the value at which the fusion occurs.

At such temperature values, the pressure due to the magnetic field is also impressive. For a particle density such as that in the atmosphere of approximately $10^{27}$ particles per 
cubic meter and for thermal energy of $10 \mathrm{keV}$, the magnetic pressure must exceed $10^{8} \mathrm{hPa}$. The field generating coils and their mechanical supports cannot withstand such pressures! To reduce the pressure it is necessary to reduce the particle density. In order to meet Lawson's plasma ignition criterion, it must be maintained under these conditions for a longer period of time.

The most efficient configuration of the magnetic field proved to be the toroidal one. The reactor chamber resembled a donut and had a closed shape like a "magnetic bottle". In fact, to ensure the stability of the plasma, the magnetic field lines follow a helical path. Such isolation is provided by devices known as Tokamak, stellarator and Reverse Pinch Field (RFP).

In a tokamak, a series of coils are placed around the torus-shaped reaction chamber. The transformer core passes through the center of the Tokamak, while the plasma current forms a secondary circuit. The perpendicular, the so-called poloidal field is induced both internally by the plasma current and externally by the poloidal coils arranged along the perimeter of the chamber.

This current heats the plasma to a very high required temperature of about 10 million $\mathrm{K}$. The idea for the tokamak came from Russian physicists Andrei Sakharov and Igor Tamm. The main disadvantage of a tokamak is the relatively narrow range of parameters. The largest Tokamak built so far is the Joint European Torus (JET).

In devices called stellarators, plasma conditions are regulated by currents flowing outside it. The helical lines of the stellar field are produced by a series of coils, which are in turn helical.

The largest star is the Large Helical Device (LHD), which began operating in 1998 at the National Fusion Research Institute in Japan. As no current is induced in the stellar plasma, heating must be obtained by other means, for example, by electromagnetic radiation. Such a technique was developed in Greifswald, Germany. These devices are similar to tokamaks in terms of toroidal and poloidal fields. However, the currents are much stronger and at the same time, the direction of the toroidal field in the plasma is reversed at the edge of the plasma. This type of system works for example in Padua, Italy.

The technique of fusion by inertial confinement consists of the preparation of a D-T pellet which is then heated rapidly to reach temperature and pressure which ensures that the plasma state is reached.

This is done when the pills are compressed by bombarding them with strong, focused laser pulses. Under these conditions, the surface of the pills evaporates and forms a crown-shaped plasma. The plasma expands and generates an inner compression front that causes the pills to implode creating an instantaneous fusion reaction.

The most advanced fusion system for inertial confinement is NOVA from the Lawrence Livermore
Laboratory in the United States. NOVA researchers have shown that densities 600 times higher than D-T liquid and 20 times higher than lead density can be achieved.

The European Community started the Joint European Torus (JET) program in 1978. The main purpose of the JET was to test fusion, plasma physics and stability conditions. Culham in Great Britain was chosen as the headquarters of JET.

The device, the largest tokamak produced to date, was put into operation in 1983 and the first controlled fusion was produced in November 1991. In 1997 a record power of $16 \mathrm{MW}$ was obtained for one second with a deuterium fuel. tritium. JET's experience has shown that a controlled merger is possible.

Its successor is the International Tokamak Experimental Reactor (ITER), an international scientific and engineering project that builds the largest nuclear tokamak fusion reactor in Cadarache, France. The ITER project aims to make the transition from experimental studies of plasma physics to large-scale energy production in fusion power plants.

The National Ignition Facility (NIF) located in California, USA is the facility with the largest and most powerful laser in the world. One of its goals is to achieve nuclear fusion and a positive energy balance for the first time - in essence, it aims to achieve a miniature star on Earth.

NIF uses very powerful lasers to heat and compresses a small amount of hydrogen-based fuel to the point where nuclear fusion reactions take place. The NIF is the largest and most powerful inertial confinement device ever built and the first to reach the regime of producing more energy than was used to ignite. Its mission is to carry out nuclear fusion in the laboratory and to support the nuclear military program by studying the behavior of matter under the conditions found inside nuclear weapons.

Extreme temperatures and pressures in the NIF target chamber will allow researchers to conduct unique experiments in high-energy-density physics and obtain new information about astrophysics such as supernovae, giant planets, or black holes.

Fusion is the opposite process of the nuclear fission reaction. In the latter, nuclei with smaller masses are obtained from a heavy nucleus and the sum of the masses produced is less than the mass of the heavy nucleus. In the case of the fusion process, the mass of the heavy nucleus is less than the sum of the initial masses of the colliding nuclei.

In order to initiate the fusion reaction, the relative energies of the colliding nuclei (positively charged particles!) Must be large enough to overcome the electrical repulsion. Therefore, in order to form helium atoms by fusing deuterium and tritium, for example, the nuclei that form the fuel must be kept under extremely high temperature and pressure conditions. 
In the mentioned reaction a neutron is produced. This neutron has a very high kinetic energy, which is released during the deceleration process. This energy can be converted into heat to produce steam, which in turn can then pass into the turbine to generate electricity. Neutrons produced in such fusion reactions can also be used to produce depleted uranium nuclear fuel, i.e., uranium containing less than ${ }^{235} \mathrm{U}$ than natural $(0.72 \%)$.

When fusion will be mastered, the planet's energy problems will be solved for a long time in a sustainable and non-polluting way (Aversa et al., 2017a-b; 2016a-b; Halliday and Robert, 1966; Kramer, 2011; Krane and Halliday, 1987; Moses et al., 2009; Petrescu, 2020a-c; 2019; 2014; 2012a-c; Petrescu and Petrescu, 2019; Petrescu et al., 2016a-d; Petrescu and Calautit, 2016a-b; Shultis and Faw, 2002).

\section{Possible Production of Neutrons from Protons by Bombarding Protons with Accelerated Electrons}

As we already said in order to discuss in more detail the fusion of two hydrogen protons, it could be interesting to study more widely the quarks inside the proton and other elementary particles with which they can interact. Protons are subatomic particles in the nuclei of all atoms, with mass $\mathrm{mp}=1,673 \cdot 10^{-27} \mathrm{~kg}$ and positive electric charge $\mathrm{qp}=\mathrm{e}=1,602 \cdot 10^{-19} \mathrm{C}$ (Fig. 1).

The number of protons is characteristic of all atoms of an element chemical. It represents the number of nuclear charges $\mathrm{Z}$ (the number of positive electrical charges). The number of protons determines the position of the element in Mendeleev's periodic system: The number of protons $=$ the number of nuclear charges $=$ the order number. The proton is symbolized by $\mathrm{p}+$.

Because all the protons of an atom have a positive charge and are all in the nucleus, the question arises why they do not repel, a common physical phenomenon in particles with the same sign of electric charge. The answer is given by the quantum field theory: Protons interact not only by electrostatic force but also by strong nuclear forces. The latter is transmitted by gluons.

Protons were discovered in 1919 by physicist Ernest Rutherford.

The problem of defining the radius of a nucleus is similar to the problem of atomic radius, in the sense that neither atoms nor their nucleus has clear delimitations. However, the nucleus can be represented as a positive charge sphere to analyze the results of electron beam scattering experiments. Because the nucleus has no welldefined limits, electrons "see" a series of effective sections that can be considered an average.

A proton (Fig. 1) is composed of three quarks, two up and one down. Each up quark has a positive charge of $2 / 3 \mathrm{e}+$ and the down quark has a negative charge of $1 / 3 \mathrm{e}-$.

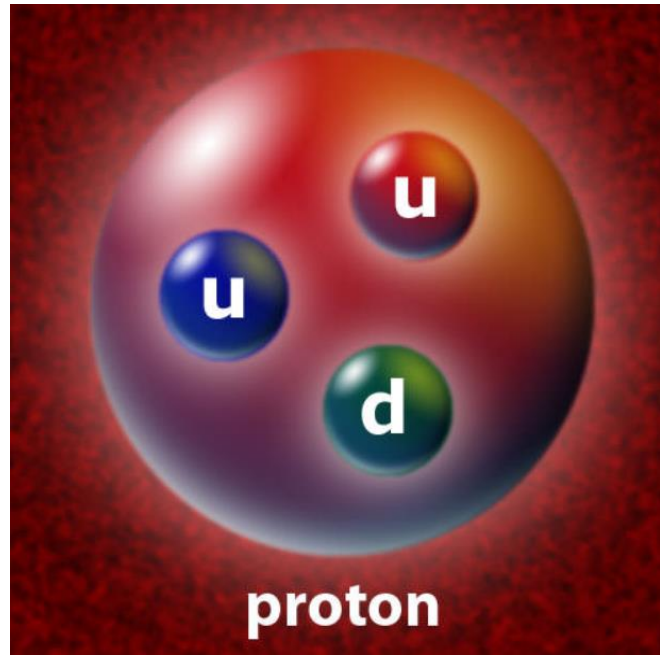

Fig. 1: A proton

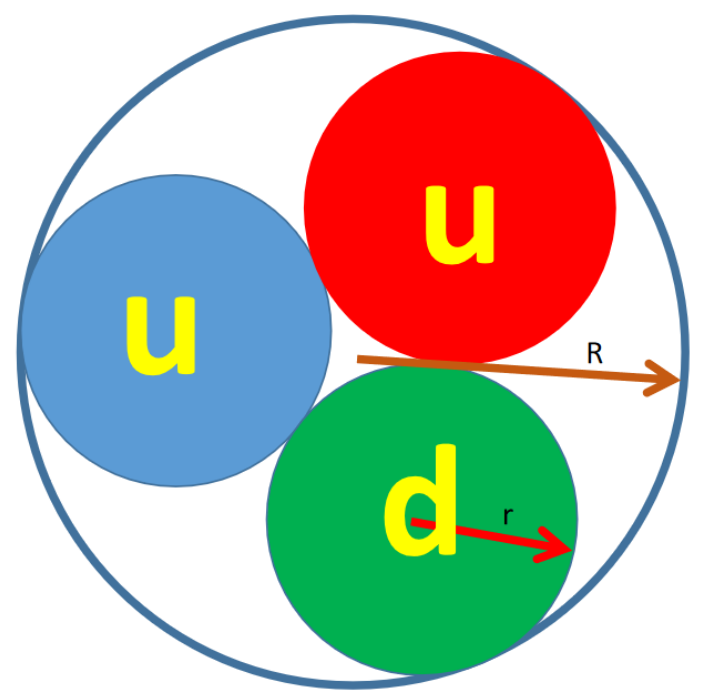

Fig. 2: A proton imagined by the authors

The most logical thing is to position the three quarks of a proton glued together, with no space between them, as we noticed that the whole world of particles is constituted, similar to those in Fig. 2, in which case between the radius of a quark $r$ and that of the proton $R$ appears relation (1):

$r=(2 \cdot \sqrt{3}-3) \cdot R$

The minimum kinetic energy required for an accelerated electron to penetrate the proton to join the down quark (negative as a charge) is calculated by the relation (2), Fig. 3, so that the accelerated electron can stick even to the negative quark, at a distance $r$ from it, considering the radius of the electron $r_{e}$ being negligible in relation to that of a quark, $r$ : 


$$
\begin{aligned}
& U_{\min }[J]=\frac{1}{4 \pi \cdot 8.8541853 E-12} \cdot \\
& \frac{(-) 1.602 E-19 \cdot(-) \frac{1}{3} 1.602 E-19}{(2 \sqrt{3}-3) \cdot R}
\end{aligned}
$$

It results immediately from relation (2) relation (3) which shows the value of the product between the minimum kinetic energy Umin (of electron acceleration, given in $\mathrm{J}$ ) and the proton radius $R$, in $\mathrm{m}$.

$$
U_{\min }[J] \cdot R[m]=\frac{1.602^{2} E-26}{12 \pi \cdot(2 \sqrt{3}-3) \cdot 8.8541853}
$$

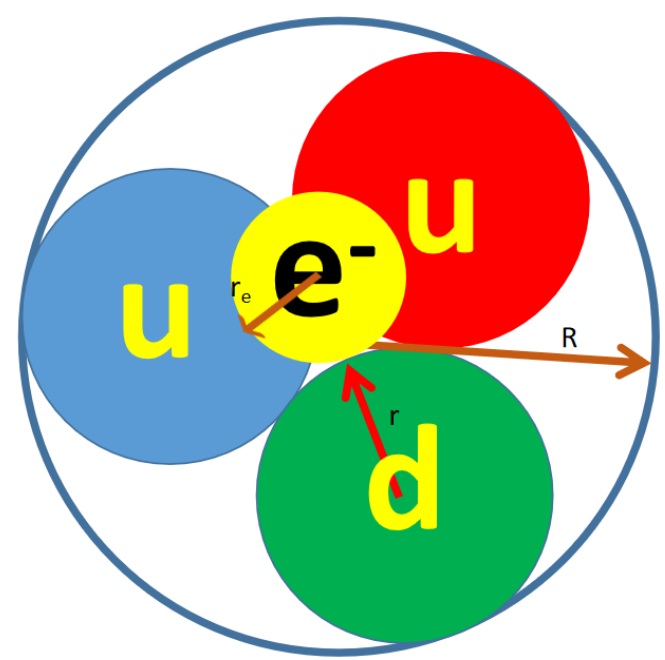

Fig. 3: A proton imagined by the authors penetrated by an electron accelerated until the limit to that, the electron can be a neighbor with the negative down quark

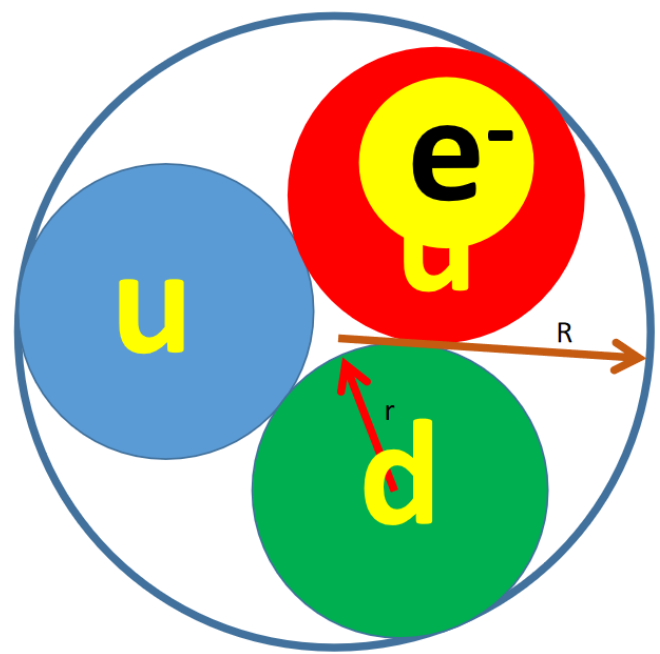

Fig. 4: Under these conditions the electron can penetrate an up quark, which become a down quark
It is more convenient to express the minimum kinetic energy required in $\mathrm{eV}$, the relation (3) thus acquiring the form (4):

$U_{\min }[e V] \cdot R[m]=\frac{1.602^{2} \cdot 6.242 E-8}{12 \pi \cdot(2 \sqrt{3}-3) \cdot 8.8541853}$

Under these conditions the electron can penetrate an up quark (Fig. 4) and transform it into a down quark (Fig. 5), so that the proton practically becomes a neutron.

Some papers present at the end a table with the normal dimensions of the proton radius at its various speeds, dimensions that are between $1 \mathrm{E}-16$ and $1 \mathrm{E}-17$ [m].

Using the equation (4) one obtains the values of the accelerated electron minimal kinetic energy $\left(U_{\min }\right)$ which may transform a proton into a neutron:

$$
\begin{aligned}
& \mathrm{R}=1 \mathrm{E}-016[\mathrm{~m}], \mathrm{E}_{\mathrm{c}}=\mathrm{U}_{\text {mine }}=10.3408439079[\mathrm{MeV}] \\
& \mathrm{R}=1 \mathrm{E}-017[\mathrm{~m}], \mathrm{E}_{\mathrm{c}}=\mathrm{U}_{\text {mine }}=103.4084390786[\mathrm{MeV}]
\end{aligned}
$$

It is easy to see that an accelerated electron can penetrate a proton in order to transform it into a neutron if the minimum kinetic energy of the penetrating by the accelerating electron is between the values of $(10-103[\mathrm{MeV}])$.

The observation is extremely important and useful in the processes of industrial fusion of matter.

About one in every 6,000 hydrogen atoms around us (including hydrogen in water) is a deuterium atom. This abundance motivates research to design fusion reactors fuel resources would be provided for billions of years!

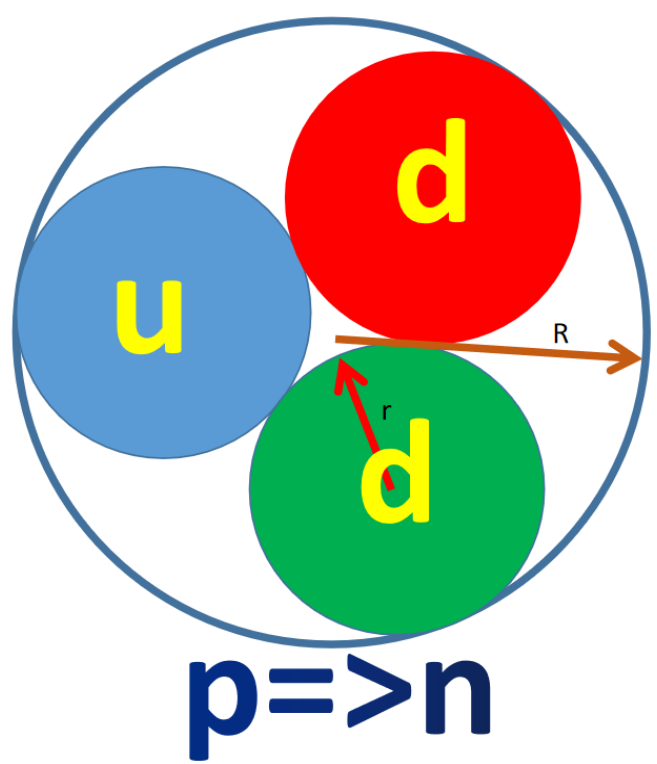

Fig. 5: Under these conditions the electron can penetrate an up quark, which become a down quark, so that the proton practically becomes a neutron 
For comparison: In order to produce $1 \mathrm{GW}$-year, about 35 tons of $\mathrm{UO}^{2}$ is needed in the case of fission and about $100 \mathrm{~kg}$ of deuterium and $150 \mathrm{~kg}$ of tritium for fusion.

Another aspect that makes fusion more attractive is the almost complete absence of any radioactive residue. In particular, no material that could be used for the production of nuclear weapons will result from the operation of such a fusion power plant. Also, unlike nuclear reactors, in which fission is used, an explosion of the plant is practically impossible: If an explosion occurs, the plasma would start to expand and cool, thus stopping the fusion process.

But this does not mean that there are no risks associated with fusion reactors. In particular, the mass production of neutrons and tritium, which is radioactive, must be taken into account. The presence of molten lithium salts and carcinogenic beryllium can also be a problem.

As in fission reactors, quite a lot of ionizing radiation (neutrons, in particular) is produced. Therefore, an important issue related to the protection against radioactivity induced throughout the installation is expected.

A possible accident in the magnetic system, which stores extremely large amounts of energy, must be seriously considered. In 1992 a team called the European Safety Assessment of Environmental and Merger Power (SEAFP) was formed.

The aim of the team is to comment on the design of nuclear fusion power plants, on the safety conditions and to assess their impact on the environment. According to SEAFP assessments, the main advantages of fusion compared to fission are that in the worst-case scenario of an accident, the radiation emission produced will not cause the population in the area to be displaced. In addition, radioactive waste produced in fusion plants decomposes relatively quickly and does not require isolation from the environment.

A possible problem is related to the release of radioactive tritium into the environment. This radioactive gas is very penetrating, dissolves easily in water and can act for a long time (the half-life of tritium is about 12 years).

It is important to check if the size of the accelerated electron corresponds to the proton so that the electron can approach the proton and have the possibility to slip right inside it to turn it into a neutron.

We use Equation (5) to determine on Beta the ratio between the speed of the accelerated electron and the speed of light, with the help of the minimum necessary kinetic energy, $103 \mathrm{MeV}$ and the rest mass of the electron $m_{0}$, where $c$ represents the speed of light in vacuum:

$$
\beta^{2}=-\frac{2 \cdot E_{c}^{2}}{c^{4} \cdot m_{0}^{2}}+\frac{2 \cdot E_{c}}{c^{2} \cdot m_{0}} \cdot \sqrt{1+\frac{E_{c}^{2}}{c^{4} \cdot m_{0}^{2}}}
$$

A corresponding minimum value of 0.9999969468 is obtained for Beta, with the help of which from the corresponding table, we extract the approximate value of the accelerated electron radius as being $8 \mathrm{E}-16[\mathrm{~m}]$, a value close to that of the proton (1E-16 [m]), but slightly higher than this, but this value corresponds to the minimum kinetic energy required to accelerate the electron, so we can increase this value accordingly so that the electron radius continues to fall below the value of the proton and even below the radius of a quark inside the proton, in order faster penetration.

We do not want to further develop other calculations on this topic because only the experiment used together with the theory proposed in this study will speak for itself.

Observation. In the presented calculations we used the hypothesis in which the accelerated electron has smaller dimensions than those of the proton it penetrates, the electron being even smaller than a quark, although, in reality, the electron even at high speeds when condensed has relatively equal dimensions to proton or at least its quark. In this way, the accelerated energy of the electron becomes sufficient and covering.

However, if the acceleration of the electron meant to penetrate a proton (in its accelerated turn) is considered, the acceleration energy required will be much higher: $\mathrm{E}_{\mathrm{c}}=$ $\mathrm{U}_{\text {mine }}[\mathrm{GeV}]=1.3479735 \approx 1.35$. In this particular case where the protons hit by the accelerated electron are also in accelerated motion with very high energies, the penetration electron must be accelerated with very high energies, very difficult to obtain, at least in circular accelerators.

\section{Materials and Methods}

The minimum fusion condition between an accelerated proton and a neutron will be presented initially at a minimum kinetic energy necessary to start the fusion between the two main elementary particles.

If the two close particles meet the plus quarks, it will be necessary to join three up quarks, so the distance between the up quarks of the proton and the neutron will be that between two positive quarks glued together (Fig. 6).

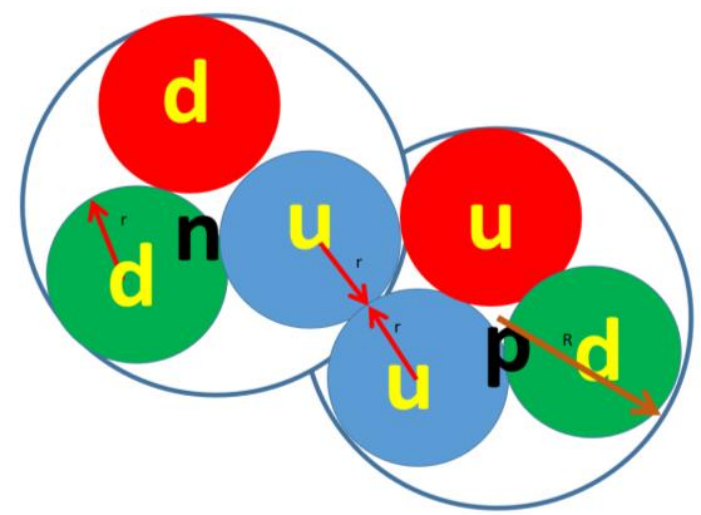

Fig. 6: A proton and a neutron in the fusion position 
In this case the minimum distance between proton and neutron would be approximately equal to that between two quarks up, $d=2 . r_{q u}$, with $r_{q u}=(2 \cdot \sqrt{3}-3) \cdot R$, where $\mathrm{R}$ is the proton radius.

The repulsion voltage between the neutron and the proton is equal to that between the three quarks up and can be written as the relation (1):

$$
\begin{aligned}
& U_{\min }[J]=E_{c \min }=\frac{1}{4 \pi \varepsilon_{0}} \cdot \frac{q_{2 q u} \cdot q_{q u}}{d}= \\
& \frac{1}{4 \pi \cdot 8.8541853 E-12} \cdot \frac{\frac{4}{3} \cdot 1.602 E-19 \cdot \frac{2}{3} \cdot 1.602 E-19}{2 \cdot(2 \sqrt{3}-3) \cdot R_{p}}
\end{aligned}
$$

The minimum kinetic energy relation (2) required to accelerate the proton will continue to be used:

$$
E_{c \min }[J]=\frac{c^{2} \cdot m_{0 p}}{2} \cdot \frac{\beta^{2}}{\sqrt{1-\beta^{2}}}
$$

By identifying the minimum kinetic energy of proton acceleration from the two relations already expressed $(1+2)$, the necessary dynamic value of the accelerated proton radius $R_{p}$ (3) can be expressed:

$$
\begin{aligned}
& R_{p}[m]=\frac{\sqrt{1-\beta^{2}}}{\beta^{2}} . \\
& \frac{2 \cdot(1.602)^{2} \cdot 10^{-26}}{c^{2} \cdot m_{0 p} \cdot 9 \cdot \pi \cdot 8.8541853 \cdot(2 \sqrt{3}-3)} \\
& =\frac{\sqrt{1-\beta^{2}}}{\beta^{2}} \cdot 2.9387 \cdot 10^{-18}
\end{aligned}
$$

The value of the dynamic radius of the proton accelerated as a function of beta can also be expressed by the distinct relation (4) known from other previous works (where beta represents the ratio between the speed of the accelerated proton $v$ and the speed of light in vacuum $c, h$ is the Planck's constant, $p$ is the Pythagoras' constant and $\mathrm{m}_{0 \mathrm{p}}$ is the rest mass of the proton):

$$
R_{p}[m]=\sqrt{\frac{10}{8}} \cdot \frac{h \cdot \sqrt{1-\beta^{2}} \cdot \sqrt{1-\frac{1}{2} \beta^{2}-\sqrt{1-\beta^{2}}}}{\pi \cdot m_{0 p} \cdot c \cdot \beta}
$$

The expressions of the accelerated proton radius from both original relations presented $(3+4)$ must generate the same values depending on the beta value and for this reason, we identify the two relations already expressed $(3+4)$ and we obtain an Equation (5) in beta:

$$
\beta \cdot \sqrt{1-\frac{1}{2} \beta^{2}-\sqrt{1-\beta^{2}}}=0.006249188
$$

\section{Results and Discussion}

To solve Equation (5) in beta we initially arrive at a fourth-degree equation, which admits several real roots, from which we choose the corresponding one, i.e., the one that satisfies both equations from which we started ( 3 and 4 ) simultaneously: Beta $^{2}=0.067080878 \Rightarrow$ Beta $=0.258999764$.

Correspondingly we obtain by both Equations ( 3 and 4) the proton radius value for the minimum accelerated proton, as being $R_{p}[\mathrm{~m}]=4.2314 \mathrm{E}-17$.

The final proton acceleration velocity, $v$ will have the value, $v_{p}[\mathrm{~m} / \mathrm{s}]=0.77646186885 \mathrm{E} 08=0.777 \mathrm{E} 08$.

The minimum kinetic energy for proton acceleration will be $E_{\text {cminp }}[\mathrm{J}]=5.22 \mathrm{E}-12$ or $E_{\text {cminp }}[\mathrm{MeV}]=32.6$.

This minimum energy of acceleration of protons that will hit neutrons, or vice versa, or both simultaneously (33 [MeV]), is much less than that required to accelerate protons when they are about to collide with each other (1.3-3 [GeV]).

Protons are also accelerated because neutron acceleration methods are more technically difficult at the moment, but accelerated neutrons obtained from the classical fusion processes already described in this study could be used because they already have half the necessary acceleration energy, 14-16 [MeV], compared to the required $32[\mathrm{MeV}]$. It is known that neutrons have a high chance of starting nuclear reactions when they are accelerated to the required energy value.

\section{Conclusion}

The paper provides researchers or theoretician an exact tool for calculating the parameters of elemental, atomic and nuclear particle.

The paper aims to present a new theoretical tool capable of helping specialists in the field of nuclear engineering to develop technological systems capable of initiating nuclear reactions and or thermonuclear in good efficiency, on an industrial scale.

The basic ideas of the paper are to obtain fusion nuclear energy by striking accelerated neutrons and protons at a certain required energy or to obtain nuclear energy by using accelerated protons, possibly together with properly accelerated electrons so that they can penetrate already accelerated protons and they also transform them into accelerated neutrons so that although the fuel used will be only simple ionized hydrogen (accelerated hydrogen protons) accelerated neutrons also appear in the system that can improve the efficiency of the nuclear fusion reaction.

\section{Acknowledgement}

This text was acknowledged and appreciated by Dr. Veturia CHIROIU Honorific member of Technical 
Sciences Academy of Romania (ASTR) Ph.D. supervisor in Mechanical Engineering.

\section{Funding Information}

Research contract: Contract number 36-5-4D/1986 from 24IV1985, beneficiary CNST RO (Romanian National Center for Science and Technology) Improving dynamic mechanisms internal combustion engines.

!All these matters are copyrighted!

Copyrights:

1. New Aircraft (New Ionic or Beam Engines): no. 548 of 22-04-2010 [cgiywDssin], Aerospace Engineering

2. Some Few Specifications About the Doppler Effect to the Electromagnetic Waves: 636 of 28-05-2010 [iEtcaouxxA], physics

3. Presenting an Atomic Model and Some Possible Applications in LASER Field: nr. 639 of 29-052010 [yncngrotfo], physics

4. Some Applications in LASER Field: no. 718 of 0907-2010 [xeujouincC], physics

5. The Energies of Today and Tomorrow: nr. 819 of 30-09-2010 [kbHquxwykr], energy engineering

6. Obtaining Energy by the Annihilation of the Matter with Antimatter - The Battle for Energy: nr. 1068 of 13.03.2011 [GfEqpGDzeh], Energy Engineering

\section{Author's Contributions}

All the authors contributed equally to prepare, develop and carry out this manuscript.

\section{Ethics}

This article is original and contains unpublished material. Authors declare that are not ethical issues and no conflict of interest that may arise after the publication of this manuscript.

\section{References}

Aversa, R., Parcesepe, D., Petrescu, R. V. V., Chen, G., Petrescu, F. I. T., Tamburrino, F., \& Apicella, A. (2016a). Glassy amorphous metal injection molded induced morphological defects.

Aversa, R., Tamburrino, F., Petrescu, R. V., Petrescu, F. I., Artur, M., Chen, G., \& Apicella, A. (2016b). Biomechanically inspired shape memory effect machines driven by muscle like acting NiTi alloys. American Journal of Applied Sciences, 13(11), 1264-1271.

Aversa, R., Petrescu, R. V., Apicella, A., \& Petrescu, F. I. (2017a). Nano-diamond hybrid materials for structural biomedical application. American Journal of Biochemistry and Biotechnology, 13(1), 34-41.
Aversa, R., Petrescu, R. V., Kozaitis, S., Apicella, A., \& Petrescu, F. I. (2017b). Some proposed solutions to achieve nuclear fusion. American Journal of Engineering and Applied Sciences, 10(3).

Halliday, D., \& Robert, R. (1966). Physics, Part II. 1st (Edn.).

Kramer, D. (2011). DOE looks again at inertial fusion as a potential clean-energy source. Physics today, 64(3), 26.

Krane, K. S., \& Halliday, D. (1987). Introductory nuclear physics.

Moses, E. I., Boyd, R. N., Remington, B. A., Keane, C. J., \& Al-Ayat, R. (2009). The National Ignition Facility: Ushering in a new age for high energy density science. Physics of Plasmas, 16(4), 041006.

Petrescu, F.I.T., 2014. Nuclear fusion. Infinite Energy, 20: 44-47.

Petrescu, F. I. (2012a). Cold Nuclear Fusion: Germany 2012. BoD-Books on Demand.

Petrescu, F. I. T. (2012b). Cold Nuclear Fusion. USA, Create Space Publisher, pp: 80.

Petrescu, F. I. T., (2012c). A New Atomic Model. Books On Demand, pp: 62.

Petrescu, F. I. T. (2019). About the nuclear particles' structure and dimensions. Computational Particle Mechanics, 6(2), 191-194.

Petrescu, F. I. T. (2020a). What is Better for Fusion? American Journal of Applied Sciences, 17(1), 83-87.

Petrescu, F. I. (2020b). A Hypothesis Which Supports the Possibility of the Existence of Dark Matter with Negative Mass. American Journal of Applied Sciences, 17, 1-5.

Petrescu, F. I. (2020c). Presents Some Aspects Related to the Atom and Atomic Electrons, Necessary in Understanding Chemical Bonds and Nanotechnologies. Necessary in Understanding Chemical Bonds and Nanotechnologies (May 24, 2020).

Petrescu, F. I. T., \& Petrescu, R. V. V. (2019). Nuclear hydrogen structure and dimensions. International Journal of Hydrogen Energy, 44(21), 10833-10837.

Petrescu, R., Aversa, R., Shuhui, L., Chen, G., APICELlA, A., Mirsayar, M., \& Petrescu, T. (2016a). Something about electron dimension.

Petrescu, F. I. T. A., Apicella, A., Aversa, R., Petrescu, R. V., Calautit, J. K., Mirsayar, M. M., \& Riccio, A. (2016b). Something about the mechanical moment of inertia.

Petrescu, R. V., Aversa, R., Apicella, A., Berto, F., Li, S., \& Petrescu, F. I. (2016c). Ecosphere protection through green energy. American Journal of Applied Sciences, 13(10), 1027-1032. 
Petrescu, F. I., Apicella, A., Petrescu, R. V., Kozaitis, S., Bucinell, R., Aversa, R., \& Abu-Lebdeh, T. (2016d). Environmental protection through nuclear energy. American Journal of Applied Sciences, 13(9), 941-946.

Petrescu, F. I., \& Calautit, J. K. (2016a). About nano fusion and dynamic fusion. American Journal of Applied Sciences, 13(3).

Petrescu, F. I., \& Calautit, J. K. (2016b). About the light dimensions. American Journal of Applied Sciences, 13(3).

Shultis, J. K., \& Faw, R. E. (2002). Fundamentals of nuclear science and engineering.

\section{Nomenclature}

$\Rightarrow$ The Planck constant: $\mathrm{h}=6.626 \mathrm{E}-34$ [Js]

$\Rightarrow$ Electrical elementary load: qe $=$ $1.6021 \mathrm{E}-19[\mathrm{C}] \mathrm{qp}=+1.6021 \mathrm{E}-19[\mathrm{C}]$

c $=$ The light speed in vacuum: $\mathrm{c}=2.997925 \mathrm{E}+08[\mathrm{~m} / \mathrm{s}]$

$$
\begin{aligned}
& \text { The permissive constant (the permittivity): } \\
& \varepsilon_{0}=8.85418 \cdot 10^{-12}\left[\frac{C^{2}}{N \cdot m^{2}}\right] \\
& \mathrm{n}=\text { The principal quantum number (the } \\
& \text { Bohr quantum number); } \\
& \mathrm{Z}=\text { The number of protons from the }
\end{aligned}
$$

\section{Source of Figures:}

Fig. 1

https://www.universetoday.com/56013/proton-parts/

Fig. 2-5

Made by authors. 